\title{
Cohort-specific imputation of gene expression improves prediction of warfarin dose for African Americans
}

\author{
Assaf Gottlieb ${ }^{1 *}$ (D), Roxana Daneshjou2, Marianne DeGorter ${ }^{2,3}$, Stephane Bourgeois ${ }^{4}$, Peter J. Svensson ${ }^{5}$, \\ Mia Wadelius ${ }^{6}$, Panos Deloukas ${ }^{4,7}$, Stephen B. Montgomery ${ }^{2,3}$ and Russ B. Altman ${ }^{2,8}$
}

\begin{abstract}
Background: Genome-wide association studies are useful for discovering genotype-phenotype associations but are limited because they require large cohorts to identify a signal, which can be population-specific. Mapping genetic variation to genes improves power and allows the effects of both protein-coding variation as well as variation in expression to be combined into "gene level" effects.

Methods: Previous work has shown that warfarin dose can be predicted using information from genetic variation that affects protein-coding regions.

Here, we introduce a method that improves dose prediction by integrating tissue-specific gene expression. In particular, we use drug pathways and expression quantitative trait loci knowledge to impute gene expression—on the assumption that differential expression of key pathway genes may impact dose requirement. We focus on 116 genes from the pharmacokinetic and pharmacodynamic pathways of warfarin within training and validation sets comprising both European and African-descent individuals.

Results: We build gene-tissue signatures associated with warfarin dose in a cohort-specific manner and identify a signature of 11 gene-tissue pairs that significantly augments the International Warfarin Pharmacogenetics Consortium dosage-prediction algorithm in both populations.

Conclusions: Our results demonstrate that imputed expression can improve dose prediction and bridge populationspecific compositions.

MATLAB code is available at https://github.com/assafgo/warfarin-cohort
\end{abstract}

Keywords: Pharmacogenomics, Warfarin dose, International Warfarin Pharmacogenetics Consortium, African Americans

\section{Background}

A crucial component to implementing precision medicine is elucidating how genetic variation affects drug response. These gene-drug associations can then be used for tailored drug selection and drug dosing [1, 2]. Genome-wide association studies (GWAS) allow the association of genetic variants like single nucleotide polymorphisms (SNPs) with a drug phenotype. While GWAS have successfully identified thousands of genotype-phenotype associations, they suffer from three limitations [3]: testing a

\footnotetext{
* Correspondence: assaf.gottlieb@uth.tmc.edu

${ }^{1}$ School of Biomedical Informatics, University of Texas Health Center, 7000

Fannin St., Houston, TX 77030, USA

Full list of author information is available at the end of the article
}

large number of SNPs requires a large study cohort to identify a statistically significant signal; SNPs can be population-specific and findings from one population may not be applicable to another population [4]; and finally, finding the genes affected by phenotypeassociated SNPs is challenging $[5,6]$.

Approaches that aggregate SNPs into genes or pathways have been developed to circumvent some of these drawbacks $[7,8]$. Working within the gene or pathway level typically decreases the number of hypotheses [5] and may also bridge population-specific allelic compositions. Beyond direct measurement of genetic variation, approaches for using measured or imputed gene expression can potentially provide insight into biological 
mechanism [9]. For example, PrediXcan [10] imputes the expected baseline expression of a gene based on the allele composition of SNPs in proximity to that gene (cis-SNPs) and uses these predicted baseline expression values to identify gene associations to disease phenotypes.

One mechanism through which SNPs may affect drug response is by modulating the expression level of genes that are key for drug response. These SNP effects may be population- and/or tissue-specific, and the GenotypeTissue Expression (GTEx) datasets [11] make it possible to assess tissue-specific baseline levels of gene expression for specific ancestries. In this work, we evaluate the degree to which estimation of baseline gene expression can improve prediction of drug response. We use the generic PrediXcan strategy in a modified way: (1) we impute gene expression in a manner that is cohort-specific; and (2) we impute genes only in tissues where expression quantitative loci (eQTLs) are associated with these genes. In order to have a more interpretable model, we focus on drug pathway genes relevant to the pharmacologic problem (see also [12]). We impute gene expression in specific tissues for each individual using the GTEx compendium [11] (Fig. 1) and learn a signature that is predictive of warfarin dose comprising genetissue pairs on a training cohort. We demonstrate the utility of the signatures by predicting warfarin dose in individuals of African American (AA) and European (EUR) descent. Warfarin dose prediction in African Americans is especially challenging, as the currently known genetic variations predict only a small amount of the dose variability $[13,14]$. In both populations, our new signatures explain the additional $8-12 \%$ of the variance in warfarin dose that is unexplained by the International Warfarin Pharmacogenetics Consortium (IWPC) algorithm. We also associate 20 novel genes with warfarin dose, including two genes-LGALS2 and PLCG2-that are common to signatures of EUR and AA populations.
Our method performs well on AA in contrast to the generic strategy. Through these improvements, we offer a general approach for prediction of drug response and discovery of associated gene candidates.

\section{Methods}

Drug response is a complex phenotype and is regulated by multiple genes and across multiple tissues. To identify which genes (in the context of a tissue) might influence warfarin dosing, we: (1) imputed the expression of genes in warfarin pharmacokinetic (PK) and pharmacodynamic (PD) pathways using SNPs in proximity to each gene; (2) used the imputed gene-tissue pairs as features to learn a tissue-specific gene expression signature on the EUR or AA training cohort; and (3) validate the performance of the learned signature gene-tissue pairs on the EUR or AA validation cohorts relative to background models.

We compared a generic PrediXcan imputation of expression [10] with an approach that: (1) builds cohortspecific imputation models; and (2) imputes gene expression for each gene only in tissues where the gene expression has associated eQTLs. We learn the tissuespecific signatures on a EUR and AA cohorts and validate them by estimating warfarin dose on independent validation cohorts.

\section{Datasets}

\section{Gene-tissue expression data}

Gene expression and eQTLs associated with 42 tissues were extracted from GTEx consortium version 6 [15] (excluding cell-lines of EBV-transformed lymphocytes and transformed fibroblasts; tissue statistics are available on the GTEx portal, http://www.gtexportal.org/home/ tissueSummaryPage).

We imputed the expression of 116 genes assembled from the curated warfarin PD and PK pathways from

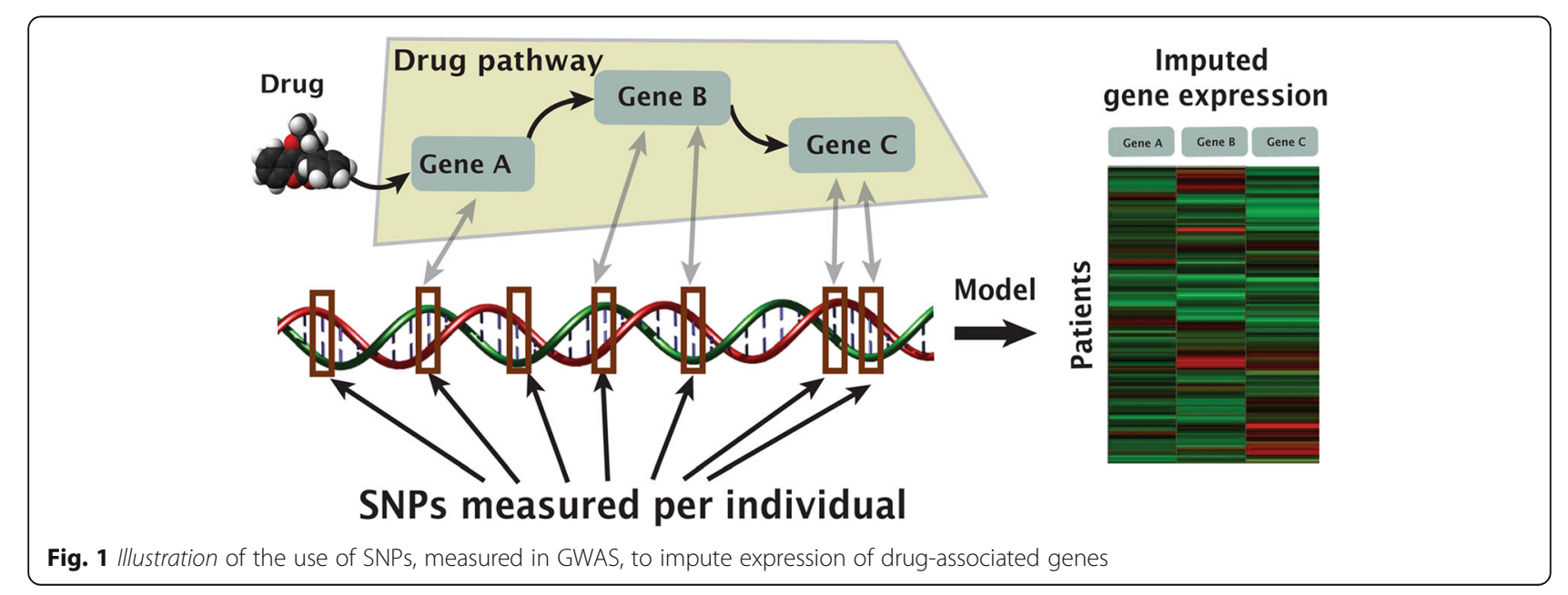


PharmGKB [16] and from the predicted warfarin PD pathway genes [17] (Additional file 1: Table S1). Prediction of warfarin PD pathway was done by connecting curated warfarin-associated genes, including gene targets, pharmacogenes (genes associated with differential drug response) and disease genes, over the protein interaction network.

\section{Training and validation cohorts}

We selected a signature comprising gene-tissue pairs, predictive of warfarin dose, using a training cohort and validated the signature performance in predicting warfarin dose on a validation cohort. Table 1 lists cohort statistics and Additional file 1: Figure S1 displays cohort age distribution. The training set for the AA signature comprised 103 previously exome-sequenced individuals of AA descent which received either low dose $(\leq 35 \mathrm{mg} /$ week) or high dose ( $>49 \mathrm{mg} /$ week) warfarin [18]. The EUR training cohort is the Cooper et al. dataset [19] with 180 genotyped individuals (five of which are of Hispanic origin), followed by imputation against HapMap. Validation was conducted on 225 genotyped individuals of AA descent, imputed against HapMap Phase 2 Release $22[13,20]$ and 233 genotyped individuals of EUR descent [21] imputed using HapMap3 (see Additional file 1: Figure S2 for distribution of doses in each study). The warfarin patient cohorts included $0.02-1.1 \%$ of missing values, which were imputed using $k$-nearest neighbors impute $(k=5)$. Based on 77 AA individuals, common to the training exome-sequenced cohort and the genotyped validation cohort, we were able to estimate $1 \%$ allelic mismatches (including missing values) between the two sequencing methods. These 77 individuals were subsequently excluded from the validation set.

\section{Imputing tissue-specific gene expression}

We imputed gene expression by building a regression model per gene in a given tissue from SNPs in cis with the gene, defined as closer than $1 \mathrm{Mb}$ to the outer bounds of the gene using the GTEx compendium of genotypes and gene expression. We built a model for each gene-tissue pair using LASSO [22], either following the original PrediXcan methodology (the "generic" strategy) [10] or a "cohort-specific" strategy. For the generic PrediXcan imputation, we used the software package of PrediXcan with the weighted cis-SNPs in the PredictDB database [23] to generate imputed genetissue pairs.

In the cohort-specific strategy, we followed the PrediXcan methodology to impute a gene using cis-SNPs (gene and SNP positions extracted from the human genome reference sequence GRCh38 and dbSNP build 144). Different from PrediXcan, we focused only on genetissue pairs where the gene has at least one significant eQTL (q-values $\leq 5 \%$ ) in GTEx [11], resulting in 67,022 SNPs measured in any of the four warfarin studies and in cis with the warfarin-pathway genes. The four imputed warfarin GWAS contain only a partial set of the SNPs available in GTEx and partial overlap with each other. To bridge these differences in SNP content, our cohort-specific strategy was to build an independent model per cohort, based only on the SNPs measured in that cohort study (Fig. 2a). The gene expression regression models where computed with LASSO [22] using fivefold cross-validation to select the optimal regularization parameters.

Between 111 and 114 genes were imputed using the generic PrediXcan and 96 genes were imputed using the cohort-specific method (Table 1). Correspondingly, the number of model SNPs is smaller with the cohort-specific method except for the AA training cohort. The AA training cohort was measured with exome-sequencing array and shares smaller number of SNPs with the genotype arrays used in GTEx. Additional file 1: Figure S3 displays the relative overlap of cis-SNPS measured in each warfarin study that were used in imputing the genes.

Our method of validation was by comparing the performance of the generic and the cohort-specific strategies in predicting warfarin dose to background models $\left(R^{2}\right.$ measures), which we describe in the next sections.

Table 1 Cohort statistics

\begin{tabular}{|c|c|c|c|c|c|c|c|}
\hline Study cohort & Patients (n) & Females (\%) & Imputation method & Model SNPs (n) & Covered tissues (n) & Imputed genes (n) & $\begin{array}{l}\text { Imputed gene-tissue } \\
\text { pairs (n) }\end{array}$ \\
\hline \multirow[t]{2}{*}{ AA training } & 103 & 68 & Generic & 1309 & 39 & 112 & 901 \\
\hline & & & Cohort-specific & 2175 & 41 & 96 & 539 \\
\hline \multirow[t]{2}{*}{ AA validation } & 225 & NA & Generic & 5384 & 39 & 114 & 1727 \\
\hline & & & Cohort-specific & 2480 & 41 & 96 & 539 \\
\hline \multirow[t]{2}{*}{ EUR training } & 180 & 36 & Generic & 5305 & 39 & 114 & 1729 \\
\hline & & & Cohort-specific & 2519 & 41 & 96 & 539 \\
\hline \multirow[t]{2}{*}{ EUR validation } & 233 & 52 & Generic & 5313 & 39 & 114 & 1729 \\
\hline & & & Cohort-specific & 2674 & 41 & 96 & 539 \\
\hline
\end{tabular}




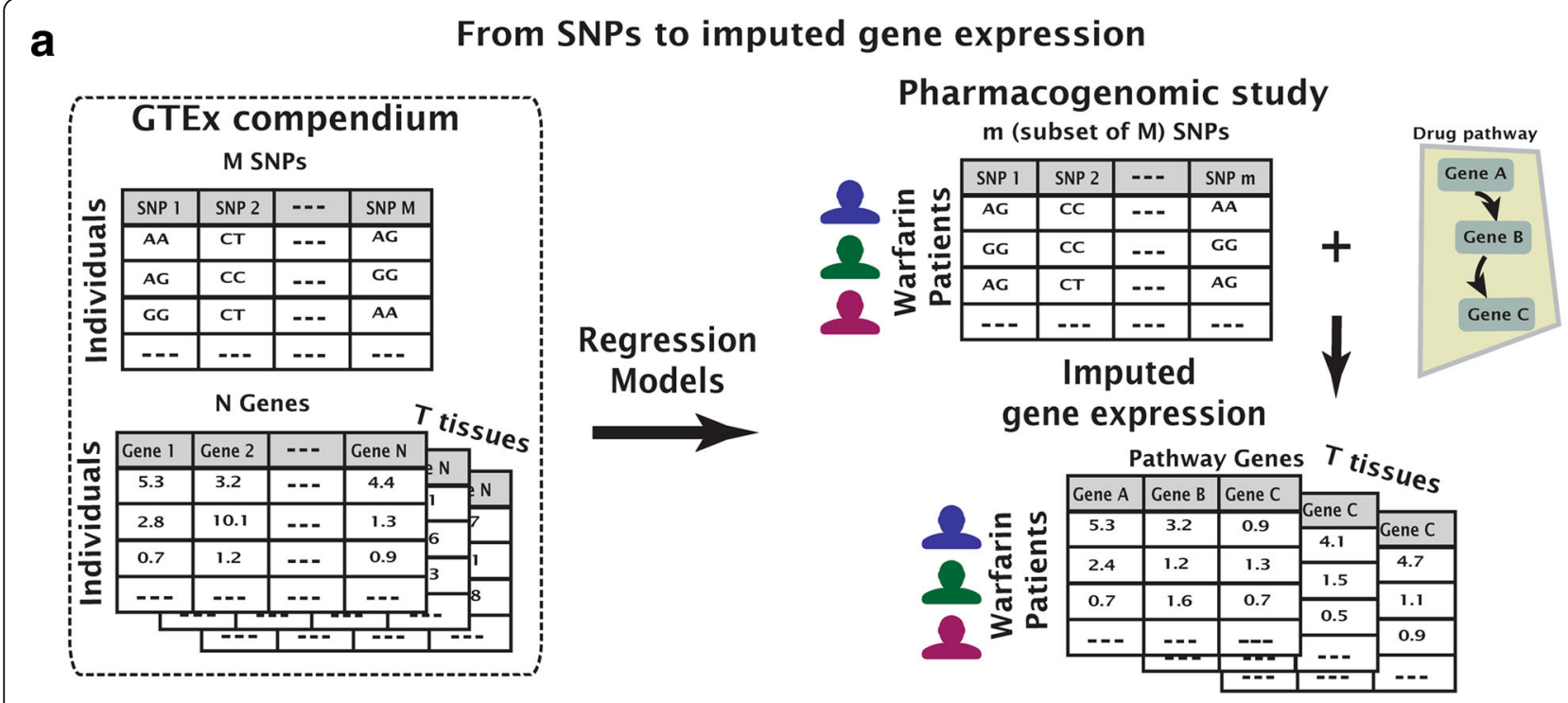

b

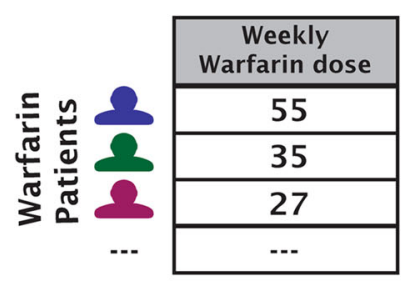

Imputed gene expression to a signature

\begin{tabular}{c|c|c|c|c|c|}
\multicolumn{4}{c}{ Liver } & \multicolumn{3}{c}{ Heart } \\
\hline Gene A & Gene B & Gene C & Gene A & Gene B & Gene C \\
\hline 5.3 & 3.2 & 0.9 & 3.6 & 4.1 & 0.1 \\
\hline 0.7 & 1.2 & 1.3 & 2.3 & 0.6 & 3.9 \\
\hline 0.7 & 1.2 & 0.9 & 7.7 & 4.1 & 6.1 \\
\hline--- &.-- &.-- &.-- &.-- &.-- \\
\hline
\end{tabular}

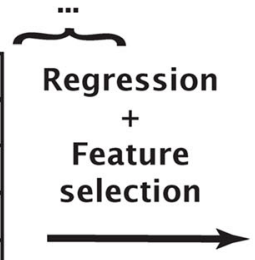

Imputed gene expression

Signature
\begin{tabular}{|c|c|}
\hline Tissue & Gene \\
\hline Liver & VKORC1 \\
\hline Esophagus & PLCG2 \\
\hline Colon & LGALS2 \\
\hline$\ldots$ & $\ldots$ \\
\hline
\end{tabular}

Fig. 2 Illustration of the feature construction and signature selection methods. First, gene expression is imputed by regression models from cis-SNPs (a). Then, a signature is learned by regressing the drug response on the imputed expression features (b)

In order to gain insights into the differences between the imputation strategies, we also compared the imputed gene-tissue expression in two ways: (1) overlap of imputed genes across the four warfarin study cohorts for each imputation method independently (between-study similarity); and (2) the consistency of each study cohort across the two imputation strategies (between-strategy similarity). For the between-study similarity, we computed the mean imputed value of each gene-tissue pair across the study individuals and computed the Pearson correlation between the gene-tissue pairs' means across the cohorts. For the between-strategy similarity, we computed the correlations of each gene-tissue pair across individual patients and averaged across all genetissue pair. All computations were performed using MATLAB v8.4. MATLAB code is available at https:// github.com/assafgo/warfarin-cohort and the imputed data in Additional file 2.

\section{Learning signatures for warfarin dose}

Imputed gene-tissue expression values were computed per warfarin patient, using all GTEx data for the 39-41 tissues, resulting in 539-1727 gene-tissue pairs serving as features (Table 1). We assume that variation in gene expression affects warfarin dose, but this effect might involve only a subset of the warfarin pathway genes and could be tissue-specific. We thus learned a signature, comprising gene-tissue pairs, that is predictive of warfarin dose using the EUR and AA training cohorts. Gene-tissue signatures were learned using LASSO regression analysis with fivefold cross-validation, selecting the shrinkage parameter which provided the minimal mean square error (Fig. 2b). For robustness, we repeated this procedure 100 times with different random crossvalidation partitions and selected for the signature genetissue pairs appearing in more than half of the repeats.

\section{Predicting warfarin dose}

We measured the performance of the signatures based on the $\mathrm{R}^{2}$ of the unexplained portion of the IWPC algorithm. Specifically, we computed the predicted dose according to the IWPC dosing algorithm for each validation cohort and inferred the residual dose, i.e. the difference between an individual's actual therapeutic 
dose and the IWPC predicted dose. In the AA validation dataset, the IWPC dose was computed without the information regarding use of enzyme reducers (phenytoin, carbamazepine, or rifampin), which could potentially change the predicted dose by up to $9 \%$ [20].

Signature performance was evaluated relative to two background models: (1) a shuffled model, in which the residual dose was shuffled 10,000 times; and (2) a random signatures model, created from 10,000 equalsized randomly selected signatures, chosen from the gene-tissue pairs not in the signature. $P$ values of regression $R^{2}$ values were empirically computed relative to these background models and corrected for false discovery rate (FDR) [24] of 0.05 across the tested signatures.

\section{Results}

We tested whether a modified PrediXcan approach could improve inference of warfarin dose for EUR and AA populations by imputing GWAS and constructing population-specific signatures. We compared the generic PrediXcan approach and the modified, cohort-specific, version based on their ability to improve the gold standard IWPC dosing algorithm.

\section{Imputing tissue-specific gene expression}

The first step involved creating features from imputed gene tissue. We assessed the differences between the two imputation strategies through between-study and between-strategy tests (see "Methods").

The between-study similarity shows the imputed gene expression distributions across studies. It was high between the EUR training and validation cohorts for both the generic and cohort-specific imputation strategies (Pearson $\rho=0.96, p \sim 0$ and $\rho=0.72, p<\mathrm{e}^{-85}$, respectively). It was significant between the AA training and validation cohorts only for the cohort-specific method (Pearson $\rho=0.54, p<2 \mathrm{e}^{-41}$, Additional file 1: Figure S4).

The between-strategy similarity displays moderate similarities between the two imputation methods-average Pearson correlations between imputed genes are in the range of $0.52-0.59( \pm 0.39)$ across the AA and EUR cohorts, with slightly higher similarities across the EUR cohorts.

\section{Learning gene-tissue signatures for warfarin dose}

We next learned separate signatures using the generic and the cohort-specific imputation strategies. The generic strategy produced a EUR signature with 16 genetissue pairs but failed to produce a signature on the AA training cohort. In contrast, the cohort-specific signatures produced a EUR signature comprising 11 gene-tissue pairs and an AA signature comprising 17 gene-tissue pairs (Table 2).

\section{Predicting warfarin dose}

We estimated the signatures performance on the validation cohorts by regressing the signature gene-tissue pairs against the residuals of the IWPC algorithm. We report here the $R^{2}$ explaining the variance of these residuals.

\section{Signatures for patients of EUR descent}

Both the generic and the cohort-specific strategies produced signatures that performed better than the background, with the generic strategy obtaining higher $\mathrm{R}^{2}$ results on the EUR validation set $\left(\mathrm{R}^{2}=0.2, p<0.008\right.$ and $\mathrm{R}^{2}=0.08, p<0.05$ for generic and cohort-specific strategies, respectively; Table 3, Fig. 3a). The cohort-specific EUR signature performed well also on the AA validation set $\left(R^{2}=0.09, p<0.02\right.$; Table 3, Fig. $\left.3 b\right)$ while the generic EUR signature was not better than the background.

\section{Signatures for patients of AA descent}

The generic imputation strategy failed to produce a signature on the AA training cohort. While the cohortspecific AA-trained signature was not better than background on the AA validation set, it showed significant improvement on both the EUR validation and EUR training cohorts $\left(\mathrm{R}^{2}=0.1, p<0.01\right.$ and $\mathrm{R}^{2}=0.24$, $p<0.0001$ for the EUR validation and training, respectively; Table 3, Fig. 3a).

\section{Analysis of gene-tissue pair signatures}

The imputed expression of three tissue-gene pairs displayed significant correlation with warfarin dose in the EUR validation cohort: (1) VKORC1 in liver and in thyroid (Pearson correlation, $\rho=0.49, p<\mathrm{e}^{-15}$ in liver and $\rho=0.28, p<\mathrm{e}^{-5}$ in thyroid); (2) STX4 in transverse colon $\left(\rho=0.3, p<2 \mathrm{e}^{-6}\right)$; and (3) CYP2C18 in liver $(\rho=0.35$, $\left.p<3 \mathrm{e}^{-8}\right)$. Out of these three, VKORC1 in liver is the only single predictor of dose that is better than the background $\left(R^{2}=0.03, p<0.005\right.$ for the cohort-specific imputed expression and $\mathrm{R}^{2}=0.03, p<0.02$ for the generic). No AA signature gene-tissue pairs displayed individually significant correlation to warfarin dose.

Three genes are common to the EUR and AA cohortspecific signatures (Table 2): VKORC1 in liver; PLCG2 in muscularis esophagus; and LGALS2 in pancreas (EUR signature) and transverse colon in (AA signature). CUBN in adrenal gland is common to the EUR generic and EUR cohort-specific signatures.

\section{Discussion}

We have introduced a strategy to use imputed gene expression in the context of drug pathways to select a signature of gene-tissue pairs predictive of warfarin dose. By focusing on drug response-associated genes, we increase the likelihood of finding biologically relevant 
Table 2 Predictive signatures for warfarin dose

\begin{tabular}{|c|c|c|c|c|c|}
\hline \multicolumn{4}{|c|}{ Cohort-specific } & \multicolumn{2}{|l|}{ Generic $^{a}$} \\
\hline \multicolumn{2}{|c|}{ African American } & \multicolumn{2}{|c|}{ Central European } & \multicolumn{2}{|c|}{ Central European } \\
\hline Gene & Tissue & Gene & Tissue & Gene & Tissue \\
\hline CYP1A1 & Adipose, subcutaneous & ARRB1 & Adipose, subcutaneous & CCND1 & Adrenal gland \\
\hline AKT1 & Pancreas & CUBN & Adrenal gland & ABL1 & Skin not-sun-exposed suprapubic \\
\hline ALOX5 & Adrenal gland; brain, cortex & GCLC & Spleen & ATF2 & $\begin{array}{l}\text { Brain, cerebellar hemisphere; brain, } \\
\text { cerebellum }\end{array}$ \\
\hline EPHX1 & Heart, left ventricle; thyroid & GGCX & Brain, cerebellar hemisphere & AURKA & Brain, frontal cortex BA9 \\
\hline GNAI2 & Whole blood & LGALS2 & Pancreas & CCND1 & Esophagus mucosa \\
\hline ITGB1 & Tibial nerve & PLCG2 & Esophagus, muscularis & CUBN & Adrenal gland \\
\hline LGALS2 & Colon transverse & PSMA6 & Skeletal muscle & CYP2C18 & Liver \\
\hline NCOA1 & Brain, caudate basal ganglia & PTK2 & Skeletal muscle & GCLM & Brain, cerebellum \\
\hline PLCG2 & Esophagus, muscularis; pancreas & UBE2I & Esophagus, muscularis & ITGA2B & Thyroid \\
\hline PROZ & Spleen & VKORC1 & Liver; thyroid & $J U N$ & Tibial artery \\
\hline SERPINF2 & Thyroid & & & MGP & Esophagus gastroesophageal junction \\
\hline SMAD3 & Esophagus, mucosa & & & PSEN1 & Pituitary \\
\hline STX4 & Colon transverse & & & SMAD2 & Testis \\
\hline VKORC1 & Liver & & & VKORC1 & Thyroid; heart atrial appendage \\
\hline
\end{tabular}

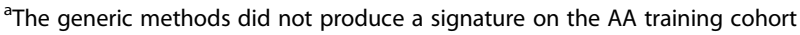

variants that influence transcriptional regulation in the context of a drug and reduce the size of the hypothesis space. We used the LASSO linear model to provide an interpretable model signature, noting that the LASSO with non-linear measurements is equivalent to one with linear measurements [25]. We compared the performance of the current state of the art, PrediXcan, to a modified, cohort-specific strategy on patients of AA and EUR descent.

We evaluated our performance by inferring the residual variation of the IWPC algorithm. The generic strategy better explained warfarin dose than our modified method on individuals of EUR descent, but performed poorly on African Americans. This is not surprising, given the preponderance of EUR-descent individuals used to generate the imputation model. Indeed, the population sampled in GTEx includes $84 \%$ white and only $14 \%$ African Americans. The results are consistent with previous findings that warfarin dose models trained on individuals of EUR descent have poor performance on African Americans [13, 26-28]. Since the AA training cohort was measured using exomesequencing while the other studies used genotyping, it is possible that these platform differences also affected the ability of the generic strategy to perform well on the AA cohort as the performance of the cohort-specific AA signature to perform better than the EUR signature on the AA validation set.

While the cohort-specific strategy performed worse on the EUR population, its signatures generalized better across populations and sequencing platforms, where EUR-trained signatures performing well on African Americans and vice versa, explaining an $\mathrm{R}^{2}$ of $0.08-0.1$ of the variability over the IWPC algorithm. Our results suggest that cohort-specific models are advantageous in cases where the set of SNPs measured for the cohort

Table 3 Performance of the generic and cohort-specific signatures on different warfarin studies

\begin{tabular}{llll}
\hline Validation cohort & Signature & $\begin{array}{l}R^{2} \text { regression against } \\
\text { IWPC residuals }\end{array}$ & $\begin{array}{l}P \text { value of difference } \\
\text { from the background }\end{array}$ \\
\hline EUR validation & EUR, generic & 0.2 & $\mathbf{0 . 0 0 8}\left(\mathbf{e}^{-\mathbf{4}}\right)$ \\
& EUR, cohort-specific & 0.08 & $\mathbf{0 . 0 0 4}(0.05)$ \\
AA validation & AA, cohort-specific & 0.1 & $\mathbf{0 . 0 0 4}(\mathbf{0 . 0 3})$ \\
\end{tabular}

The $\mathrm{R}^{2}$ coefficient is measured on the IWPC residuals

$P$ values below FDR of 0.05 are bolded

${ }^{a}$ Background computed as random signatures. $P$ values of shuffled signatures in parentheses 


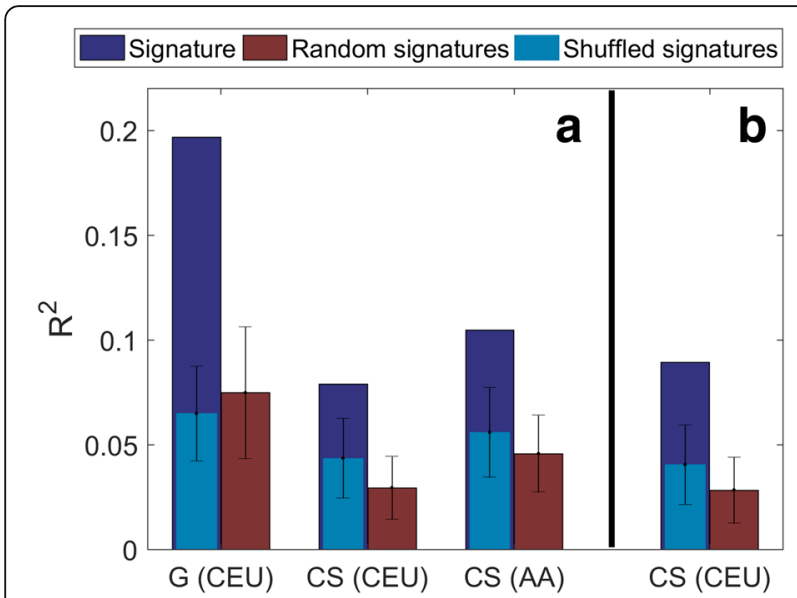

Fig. $3 R^{2}$ results of the predicted unexplained variance in warfarin dose by the IWPC algorithm for the EUR (a) and AA (b) validation cohorts. Represented are the signatures (dark blue), random signatures (red), and signatures on shuffled data (light blue) as the background models for the AA and EUR signatures. EUR and AA in parentheses are the training cohort for the signature; $G$ generic imputation method, CS cohort-specific imputation

differ from the set used for building the models and calls for diversifying the sampled populations in GTEx to produce better pharmacogenomic models. As the gender and age distributions in GTEx (34\% women and majority of individuals aged $50-70$ years) differ from the distributions in the warfarin cohorts (Table 1 and Additional file 1: Figure S1), we estimate that models that further stratify the GTEx population based on these covariates may produce more accurate imputation models and improve the pharmacogenomics models.

Warfarin acts as an inhibitor of VKORC1 and is part of the IWPC dose algorithm. Polymorphisms in its cisSNP rs9923231 account for approximately $25 \%$ of the variance in stabilized warfarin dose and is currently considered the single largest predictor of warfarin dose [29]. While the generic and cohort-specific models for VKORC1, in both the AA and EUR training cohorts, do not include this SNP, they include SNPs in linkage disequilibrium with it, such as rs2855475, rs2359612, and rs749767 $\left(R^{2}>0.96\right)$ along with $13-31$ additional SNPs in both models. It is encouraging that imputed expression of VKORC1 in liver was found to be a strong dose predictor, suggesting that a significant component in VKORC1 effect on dose is through transcriptional regulation.

Only three genes, VKORC1, STX4, and CYP2C18, are individually correlated with warfarin dose, accounting together for less than one-third of the entire signature explained dose $\left(R^{2}=0.035, p<0.02\right.$ on the EUR cohort and insignificant on the AA cohort), which suggests that gene expression associated with dose is combinatorial in nature and supports our pathway-directed multivariate analysis methodology. Specifically, STX4 resides close to VKORC1 ( $50 \mathrm{~Kb}$ apart) and some of the warfarinassociated SNPs like rs10871454 [19] could be attributed to either genes or affect both gene expression. Additionally, the cohort-specific signatures include genes with known polymorphism associated with warfarin dose such as GGCX, within African Americans [30] and EPHX1 within Caucasians [31]. Lastly, CYP2C18, appearing in the generic signature, was previously reported to be associated with warfarin dose [32]. The report explained this association by linkage disequilibrium of CYP2C18-associated SNP rs7896133 with CYP2C9*3 (PredictDB does not include a CYP2C9 model in liver). rs7896133 is indeed moderately linked to CYP2C9*3 $\left(\mathrm{r}^{2}=0.68\right.$, using SNAP tool [33]) and is included in PredictDB models for CYP2C18 in liver but the generic models include additional eight cisSNPs, four of them (rs7067881, rs9332214, rs7920801, and rs7088784) with similar or larger weights than rs7896133 and only two of these in LD with CYP2C9*3 (rs9332214 and rs7088784, $\mathrm{r}^{2}=0.87$ and 0.68 , respectively), suggesting that $\mathrm{CYP} 2 \mathrm{C} 18$ might have another mechanism of association with warfarin dose. Notably, rs12777823, associated with warfarin dose in African Americans and independent of CYP2C9*2 and CYP2C9*3 [34] was not measured in any of the four cohorts in our study and was also not part of the generic models in PredictDB.

Liver is associated with two (out of three) gene-tissue pairs correlated with warfarin dose (VKORC1 and CYP2C18). Liver indeed plays a role in the metabolism of warfarin [35]. We have not a priori filtered tissues based on estimated relevance to warfarin response. Our signatures thus include tissues that are not typically considered relevant to warfarin's mode of action. The reasons may be that these expression values in the selected tissues may correlate with expression in other relevant tissues or may represent genuine evidence of an unexpected role of new tissues in warfarin response. Tissuespecific knowledge may improve our methodology and should be considered in follow-up work, taking into account also tissue-specific detection sensitivity [15].

\section{Conclusions}

Imputation of gene expression for genes relevant to drug action can increase the power of GWAS in explaining drug response such as warfarin dose. Focusing on genes with genetically driven (vs environmentally driven) expression in the form of eQTLs allows us to build models of drug response that reflect the expected effects of critical genes in particular tissues. We have further shown that these models can be computed using a cohortspecific strategy to improve their predictive power. 
We chose to use previous knowledge in the form of pre-selected genes in order to avoid overfitting and for interpretability of the results. However, imputing all the genes can potentially improve the model further and identify novel genes associated with warfarin dose. For the same reason of interpretability, we used the LASSO linear regression model that results in relatively short and robust signatures, notably showing that the LASSO with non-linear measurements is in many cases equivalent to one with linear measurements [25]. Nevertheless, it would be worthwhile in future works to compare the performance of other non-linear regression models in predicting warfarin dose.

\section{Additional files}

Additional file 1: Supplementary figures and tables. (PDF $724 \mathrm{~kb}$ )

Additional file 2: A zip files containing the four imputed data files WarfarinData.zip. (ZIP $997 \mathrm{~kb})$

\section{Abbreviations}

AA: African American; eQTL: Expression quantitative loci; EUR: European; FDR: False discovery rate; GTEx: Genotype-tissue expression; GWAS: Genomewide association studies; IWPC: International warfarin pharmacogenetics consortium; PD: Pharmacodynamic; PK: Pharmacokinetic; SNP: Single nucleotide polymorphisms

\section{Acknowledgements}

We would like to thank the reviewers for helpful suggestions.

\section{Funding}

RBA and AG are funded by the NIH grants LM05652, GM102365, and GM061374. RD is funded by Stanford Medical Scientist Training Program and the Paul and Daisy Soros Fellowship for New Americans. MKD is funded by a Banting Postdoctoral Fellowship. SBM was funded by the Edward Mallinckrodt Jr Foundation and NIH grants R01MH101814, R01HG008150, U01HG007436, and U01HG00908001. PD is funded by the British Heart Foundation (BHF) grant RG/14/ $5 / 30893$ and forms part of the research themes contributing to the translational research portfolios of the Barts Biomedical Research Centre funded by the National Institute for Health Research (NIHR). MW is funded by the Swedish Heart-Lung Foundation (20120557 and 20140291) and the Swedish Research Council (Medicine 521-2011-2440 and 521-2014-3370).

\section{Availability of data and materials}

The datasets used and/or analyzed during the current study are available from the authors of the studies on reasonable request.

GTEx data (v6) was obtained from the GTEx portal and from dbGaP (phs000424.v6.p1).

\section{Authors' contributions}

AG conceived the paper, performed the experiments, and analyzed the data. $\mathrm{RD}$ and RBA contributed to the experimental design. MKD and SBM computed the eQTL associations on the GTEx data. PJS, PD, MW, and SB performed the European-descent GWAS, used here as a validation cohort. AG, RD, MKD, SBM, and RBA wrote the manuscript. All authors read and approved the final manuscript.

\section{Ethics approval and consent to participate}

The EUR training warfarin study was approved by the Human Subjects Review Committee at the University of Washington and informed consent was obtained in accordance with the Declaration of Helsinki, as described in Cooper et al. [19].

The EUR validation cohort study was approved by the Regional Ethics Committee in Lund, Sweden, and informed consent was obtained in accordance with the Declaration of Helsinki [21].
For the AA training cohort, all patients consented using an Institutional Review Board-approved process at each respective collection site in accordance with the Declaration of Helsinki, as described in Daneshjou et al. [18]. For the AA validation cohort, all participants gave written informed consent to participate and all protocols were approved by local institutional review boards, as described in Isma et al. [21].

\section{Consent for publication}

Not applicable.

\section{Competing interests}

The authors declare that they have no competing interests.

\section{Publisher's Note}

Springer Nature remains neutral with regard to jurisdictional claims in published maps and institutional affiliations.

\section{Author details}

${ }^{1}$ School of Biomedical Informatics, University of Texas Health Center, 7000 Fannin St., Houston, TX 77030, USA. ²Department of Genetics, Stanford University, Stanford, CA 94305, USA. ${ }^{3}$ Department of Pathology, Stanford University, Stanford, CA 94305, USA. ${ }^{4}$ William Harvey Research Institute, Barts and the London School of Medicine and Dentistry, Queen Mary University of London, London EC1M 6BQ, UK. ${ }^{5}$ Department of Translational Medicine, University of Lund, Malmö 205 02, Sweden. ${ }^{6}$ Department of Medical Sciences and Science for Life laboratory, Uppsala University, Uppsala 751 85, Sweden. ${ }^{7}$ Princess Al-Jawhara Al-Brahim Centre of Excellence in Research of Hereditary Disorders (PACER-HD), King Abdulaziz University, Jeddah 21589, Saudi Arabia. ${ }^{8}$ Department of Bioengineering, Stanford University, Stanford, CA 94305, USA.

Received: 16 August 2017 Accepted: 14 November 2017

Published online: 24 November 2017

\section{References}

1. Fernald GH, Capriotti E, Daneshjou R, Karczewski KJ, Altman RB. Bioinformatics challenges for personalized medicine. Bioinformatics. 2011;27:1741-8.

2. Ginsburg GS, McCarthy JJ. Personalized medicine: revolutionizing drug discovery and patient care. TRENDS in Biotechnology. 2001;19:491-6.

3. Roukos DH. Personal genomics and genome-wide association studies: novel discoveries but limitations for practical personalized medicine. Ann Surg Oncol. 2009:16:772-3.

4. Rosenberg NA, Huang L, Jewett EM, Szpiech ZA, Jankovic I, Boehnke M. Genome-wide association studies in diverse populations. Nat Rev Genet. 2010;11:356-66

5. Wang K, Dickson SP, Stolle CA, Krantz ID, Goldstein DB, Hakonarson H. Interpretation of association signals and identification of causal variants from genome-wide association studies. Am J Hum Genet. 2010;86:730-42.

6. Ritchie MD. The success of pharmacogenomics in moving genetic association studies from bench to bedside: study design and implementation of precision medicine in the post-GWAS era. Hum Genet. 2012;131:1615-26.

7. Björkegren JL, Kovacic JC, Dudley JT, Schadt EE. Genome-wide significant loci: how important are they? Systems genetics to understand heritability of coronary artery disease and other common complex disorders. J Am Coll Cardiol. 2015;65:830-45.

8. Limdi NA, Wadelius M, Cavallari L, Eriksson N, Crawford DC, Lee M-TM, et al. Warfarin pharmacogenetics: a single VKORC1 polymorphism is predictive of dose across 3 racial groups. Blood. 2010;115:3827-34.

9. Li L, Kabesch M, Bouzigon E, Demenais F, Farrall M, Moffatt MF, et al. Using eQTL weights to improve power for genome-wide association studies: a genetic study of childhood asthma. Front Genet. 2013;4:103.

10. Gamazon ER, Wheeler HE, Shah KP, Mozaffari SV, Aquino-Michaels K, Carroll $R J$, et al. A gene-based association method for mapping traits using reference transcriptome data. Nat Genet. 2015;47:1091-8.

11. GTEx Consortium. The Genotype-Tissue Expression (GTEx) pilot analysis: Multitissue gene regulation in humans. Science. 2015;348:648-60.

12. Montgomery SB, Dermitzakis ET. From expression QTLs to personalized transcriptomics. Nat Rev Genet. 2011;12:277-82. 
13. Daneshjou R, Tatonetti NP, Karczewski KJ, Sagreiya H, Bourgeois S, Drozda K, et al. Pathway analysis of genome-wide data improves warfarin dose prediction. BMC Genomics. 2013;14:S11.

14. Drozda K, Wong S, Patel SR, Bress AP, Nutescu EA, Kittles RA, et al. Poor warfarin dose prediction with pharmacogenetic algorithms that exclude genotypes important for African Americans. Pharmacogenet Genomics. 2015;25:73.

15. Ardlie KG, Deluca DS, Segrè AV, Sullivan TJ, Young TR, Gelfand ET, et al. The Genotype-Tissue Expression (GTEX) pilot analysis: Multitissue gene regulation in humans. Science. 2015;348:648-60.

16. Whirl-Carrillo M, McDonagh E, Hebert J, Gong L, Sangkuhl K, Thorn C, et al. Pharmacogenomics knowledge for personalized medicine. Clin Pharmacol Ther. 2012;92:414

17. Gottlieb A, Altman RB. Integrating systems biology sources illuminates drug action. Clin Pharmacol Ther. 2014:95:663-9.

18. Daneshjou R, Gamazon ER, Burkley B, Cavallari LH, Johnson JA, Klein TE, et al. Genetic variant in folate homeostasis is associated with lower warfarin dose in African Americans. Blood. 2014;124:2298-305.

19. Cooper GM, Johnson JA, Langaee TY, Feng H, Stanaway IB, Schwarz UI, et al. A genome-wide scan for common genetic variants with a large influence on warfarin maintenance dose. Blood. 2008;112:1022-7.

20. Perera MA, Cavallari LH, Limdi NA, Gamazon ER, Konkashbaev A, Daneshjou $\mathrm{R}$, et al. Genetic variants associated with warfarin dose in African-American individuals: a genome-wide association study. Lancet. 2013;382:790-6.

21. Isma N, Svensson PJ, Gottsäter A, Lindblad B. Prospective analysis of risk factors and distribution of venous thromboembolism in the populationbased Malmö Thrombophilia Study (MATS). Thromb Res. 2009;124:663-6

22. Tibshirani R. Regression shrinkage and selection via the lasso. J R Stat Soc B Methodol. 1996;58:267-88.

23. Wheeler HE, Shah KP, Brenner J, Garcia T, Aquino-Michaels K, Cox NJ, et al. Survey of the heritability and sparse architecture of gene expression traits across human tissues. PLoS Genet. 2016;12:e1006423.

24. Benjamini Y, Hochberg Y. Controlling the false discovery rate: a practical and powerful approach to multiple testing. J R Stat Soc B Methodol. 1995; 57:289-300.

25. Thrampoulidis C, Abbasi E, Hassibi B. Lasso with non-linear measurements is equivalent to one with linear measurements. In Advances in Neural Information Processing Systems. 2015. pp. 3420-8.

26. Scott SA, Khasawneh R, Peter I, Kornreich R, Desnick RJ. Combined CYP2C9, VKORC1 and CYP4F2 frequencies among racial and ethnic groups. Pharmacogenomics. 2010;11:781-91.

27. Roper N, Storer B, Bona R, Fang M. Validation and comparison of pharmacogenetics-based warfarin dosing algorithms for application of pharmacogenetic testing. J Mol Diagn. 2010;12:283-91.

28. Shaw PB, Donovan JL, Tran MT, Lemon SC, Burgwinkle P, Gore J. Accuracy assessment of pharmacogenetically predictive warfarin dosing algorithms in patients of an academic medical center anticoagulation clinic. J Thromb Thrombolysis. 2010:30:220-5.

29. Owen RP, Gong L, Sagreiya H, Klein TE, Altman RB. VKORC1 pharmacogenomics summary. Pharmacogenet Genomics. 2010;20:642.

30. Cavallari LH, Perera M, Wadelius M, Deloukas P, Taube G, Patel SR, et al. Association of the GGCX (CAA) 16/17 repeat polymorphism with higher warfarin dose requirements in African Americans. Pharmacogenet Genomics. 2012;22:152.

31. Liu H-Q, Zhang C-P, Zhang C-Z, Liu X-C, Liu Z-J. Influence of two common polymorphisms in the EPHX1 gene on warfarin maintenance dosage: a meta-analysis. Biomed Res Int. 2015;2015:564149.

32. Wadelius M, Chen LY, Eriksson N, Bumpstead S, Ghori J, Wadelius C, et al. Association of warfarin dose with genes involved in its action and metabolism. Hum Genet. 2007;121:23-34.

33. Johnson AD, Handsaker RE, Pulit SL, Nizzari MM, O'Donnell CJ, De Bakker PI. SNAP: a web-based tool for identification and annotation of proxy SNPS using HapMap. Bioinformatics. 2008;24:2938-9.

34. Perera M, Gamazon E, Cavallari L, Patel S, Poindexter S, Kittles R, et al. The missing association: sequencing-based discovery of novel SNPs in VKORC1 and CYP2C9 that affect warfarin dose in African Americans. Clin Pharmacol Ther. 2011;89:408-15

35. Rost S, Fregin A, Ivaskevicius V, Conzelmann E, Hörtnagel K, Pelz H-J, et al. Mutations in VKORC1 cause warfarin resistance and multiple coagulation factor deficiency type 2. Nature. 2004;427:537-41.

\section{Submit your next manuscript to BioMed Central and we will help you at every step:}

- We accept pre-submission inquiries

- Our selector tool helps you to find the most relevant journal

- We provide round the clock customer support

- Convenient online submission

- Thorough peer review

- Inclusion in PubMed and all major indexing services

- Maximum visibility for your research

Submit your manuscript at www.biomedcentral.com/submit
Biomed Central 